\title{
Fisiopatología y factores de virulencia del Streptococcus pyogenes implicados en Ia erisipela, celulitis y fascitis necrotizante
}

\section{Artículo de revisión}

\author{
Rodríguez-Berber Yahir Alejandro*, Azúa-Díaz Gerardo Gilberto**
}

\section{Resumen}

- Sin tratamiento oportuno, las lesiones cutáneas por Strepto- coccus pueden poner en peligro la vida de una persona. En el - caso de México hay factores para que se den estas infeccio- nes por el incremento en el número de enfermos con diabetes - mellitus tipo 2, que produce un estado de inmunosupresión en - el organismo. Las vías de entrada para la bacteria pueden ser - desde una tiña de los pies, un mal rasurado, hasta trauma- tismos graves. En el caso de S. pyogenes puede manifestarse - desde una erisipela o una celulitis hasta una fascitis necroti-

- zante o síndrome de shock tóxico estreptocóccico. Estas le-

- siones están relacionadas con los factores de virulencia de la

- bacteria que le permiten adherirse a la epidemis (fibronectin-

- binding, proteína M), evadir el sistema inmune (Mac-I, Mac-2,

- EndoS, SpeB, la cápsula, ScrpA) y comenzar un proceso de

- patogénesis (estreptolisina O, estreptolisina S, hialuronidasa,

- estreptocinasa, superantígenos). El objetivo de este trabajo es

- mostrar la fisiopatología de los principales factores de virulen-

- cia del Streptococo pyogenes que generan lesiones cutáneas. LUXMÉDICA AÑO 16, NÚMERO 47, MAYO-AGOSTO 2021, PP 39-50.

Palabras clave: diabetes, erisipela, celulitis, fascitis necrotizante, síndrome del shock tóxico estreptocóccico, estreptolisina, proteína $M$, estreptocinasa, superantígeno.

\section{Abstract}

Without prompt treatment, Streptococcus skin lesions can endanger a person's life. In Mexico, these infections occur due to an increased number of patients with type 2 diabetes mellitus, which produces immunosuppression in the body. The bacteria's entry routes can range from ringworm of the feet, poor shaving to severe trauma. In the case of S. pyogenes, its manifestations can range from erysipelas or cellulitis to necrotizing fasciitis or streptococcal toxic shock syndrome. These lesions are related to the virulence factors of the bacterium that allow it to adhere to the epidermis (FN-BINDING, M protein), evade the immune system (Mac-I, Mac-2, EndoS, SpeB, capsule, ScrpA), and start a pathogenesis process (streptolysin $O$, streptolysin S, hyaluronidase, streptokinase, superantigens). This article aims to review the pathophysiology of the virulence factors of Streptococcus pyogenes, which generate skin leSiOnS. LUXMÉDICA AÑO 16, NÚMERO 47, MAYO-AGOSTO 2021, PP 39-50.

Keywords: diabetes, erysipela, cellulitis, necrotizing fasciitis, streptococcal toxic shock syndrome, streptolysin, $M$ protein, streptokinase, superantigen

Estudiante de la Carrera de Medicina del Centro de Ciencias de la Salud de la Universidad Autónoma de Aguascalientes. https://orcid.org/0000-0003$3295-4936$.

Correo electrónico: yahir.berber@gmail.com

** Profesor Investigador del Departamento de Medicina del Centro de Ciencias de la Salud de la Universidad Autónoma de Aguascalientes. https://orcid. org/0000-0002-8065-3487. Correo electrónico: gerardo.azua@edu.uaa.mx

Fecha de recibido: 14 de octubre 2020

Fecha de aceptación: 13 de marzo 2021

Correspondencia: Dr. Gerardo Gilberto Azúa Díaz. Departamento de Medicina. Edificio 107, Ciudad Universitaria. Universidad Autónoma de Aguascalientes. Avenida Universidad 940. Aguascalientes, Ags., México. Código postal 20131. Teléfono 449 9108440. Correo electrónico: gerardo.azua@edu.uaa.mx 


\section{Introducción}

Streptococcus pyogenes es una bacteria gram + , con presencia de una capa de peptidoglucanos alrededor de su membrana citoplasmática. ${ }^{1}$ Entre sus principales características se encuentran la forma de coco esférico, de 1 a $2 \mu$ m de diámetro, está ordenado en cadenas cortas, no es móvil, no produce esporas, es anaerobio facultativo, catalasa negativa y produce beta hemólisis, es decir, provoca la lisis completa de los eritrocitos dando un color amarillo en la placa de agar sangre. ${ }^{2}$

S. pyogenes se encuentra en el grupo A de la clasificación de Lancefield; es decir, anclada a su capa de peptidoglucanos, tiene cadenas de $\mathrm{N}$-acetilglucosamina y ramnosa, constituyendo del $40 \%$ al $60 \%$ de la pared de la bacteria. ${ }^{3}$

De forma natural, esta bacteria se encuentra como flora transitoria en la piel y las mucosas evitando la respuesta inmune gracias a sus factores de virulencia y en personas con un sistema inmune competitivo no genera patogenia. ${ }^{4}$

Las personas que se encuentran mayormente afectadas son aquellas que se encuentran inmunocomprometidas (diabetes mellitus, VIH/Sida, asplenia, entre otros), así como las que tienen alguna vía de entrada por trauma, quemadura y que se encuentran en edades en rangos menores de 5 años y/o mayores de 60 años.,

La diabetes mellitus (DM) genera inmunosupresión a través de diversos mecanismos:

- Disminución de síntesis y liberación de interleucina 1 e interleucina 6 (IL-1, IL-6) por monocitos y macrófagos en respuesta a los lipopolisacáridos bacterianos. ${ }^{7}$

- Disminución de la expresión del complejo mayor de histocompatibilidad (MHC) clase I, en la superficie de las células mieloides. ${ }^{7,8}$

- Disminuye la quimiotaxis y la capacidad fagocítica de las células polimorfonucleares (PMN). ${ }^{7}$

- Disminuye los niveles del complemento, especialmente C4.7,8

Es importante mencionar que la DM retarda la cicatrización, lo que permite la entrada a un mayor número de patógenos, por lo que cualquier herida puede representar una vía de entrada para infecciones.

En México, la DM en los últimos 6 años ha tenido un ascenso en la tasa de crecimiento promedio (TCP) en el periodo 2014-2019 con un alza del $5.93 \%$, siendo los años 2014 a 2015 el pico de la TCP con un incremento del $33.41 \%$. Son las infecciones inespecíficas la segunda causa de ingreso hospitalario en las personas con DM (ver figura 1)..$^{9-14}$

La infección por $\mathrm{VIH}$ se ha mantenido al margen con sólo un incremento en la TCP del $0.74 \%$ del periodo 2014-2020, aunque cabe señalar que se estima que en el periodo 1983-2019 hay un 
total de casos vivos con SIDA de 87,790, mientras que los que se hayan como serotipo a VIH son un total 92,866 casos vivos representando estos dos un total de 180,656 casos totales de VIH/SIDA en México. ${ }^{15}$

El aumento constante de personas inmunocomprometidas en México (DM, VIH), puede predisponer al desarrollo de infecciones por $S$. pyogenes, estableciendo la necesidad de mejorar el conocimiento clínico, diagnóstico y terapéutico de lesiones cutá- neas producidas por este microorganismo, con el fin de mejorar el pronóstico del paciente. En cuanto a la erisipela y la celulitis no se conoce con certeza la incidencia real, no hay estudios epidemiológicos recientes en México. ${ }^{16}$ En cuanto a la fascitis necrotizante, se estima que su incidencia es de 0.4 por cada 100,000 en Estados Unidos, en México tampoco hay estudios epidemiológicos recientes. ${ }^{17}$

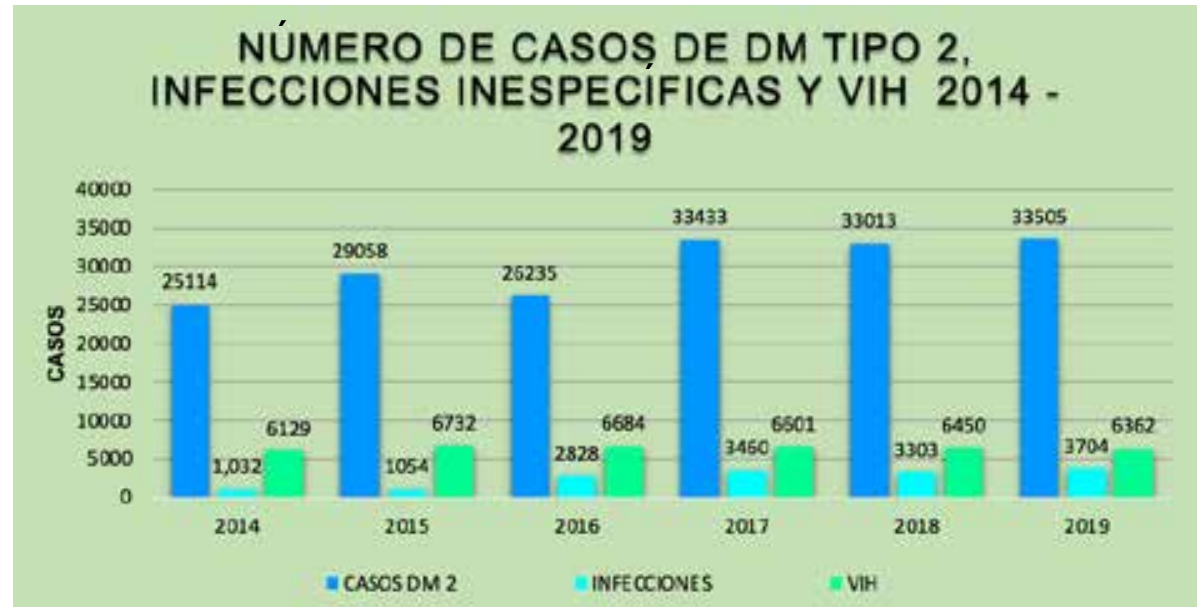

Figura 1. Casos diagnosticados por año de la Diabetes mellitus tipo 2 y el número de infecciones inespecíficas por las cuales los pacientes ingresaron a un hospital. Así como el número de casos diagnosticados por año desde 2014 de SIDA..$^{9-15}$

\section{Factores de virulencia y adherencia a la piel}

El primer paso para presentar una infección por $S$. pyogenes, es la adherencia a células epiteliales gracias a la unión con fibronectina a través de proteínas de unión de fibronectina (Fn-binding), de las cuales se conocen hasta once tipos distintos (figura 2); las Fn-bindings forman un puente con la integrina $\alpha 5 \beta 1$ presente en la fibronectina. ${ }^{18}$ Además, las proteínas $M$ son un grupo de proteínas adhesinas que se pueden anclar a una amplia gama de células. Cabe destacar que la M6 logra anclarse con el cofactor CD46 de los queratinocitos, así como la integrina $\alpha 5 \beta 1$ presente en la fibronectina; a su vez, la proteína $M$ funciona como un factor antifagocítico al impedir la maduración del fagosoma e inhibir la deposición del C3b. ${ }^{19}$

En el caso del serotipo M1 y la fibronectina del grupo $A$ estreptocóccico tipo $A$ (FbaA), activan y mandan a la célula epitelial señales para ser digerida. Por el otro lado, el serotipo M3 mediante la fibronectina del grupo A estreptocóccico tipo B (FbaB) induce la activación de las proto-oncogene tirosina-proteína quinasa ( $\mathrm{Src}$ ) permitiendo la entrada de la bacteria a la célula. ${ }^{20}$ 


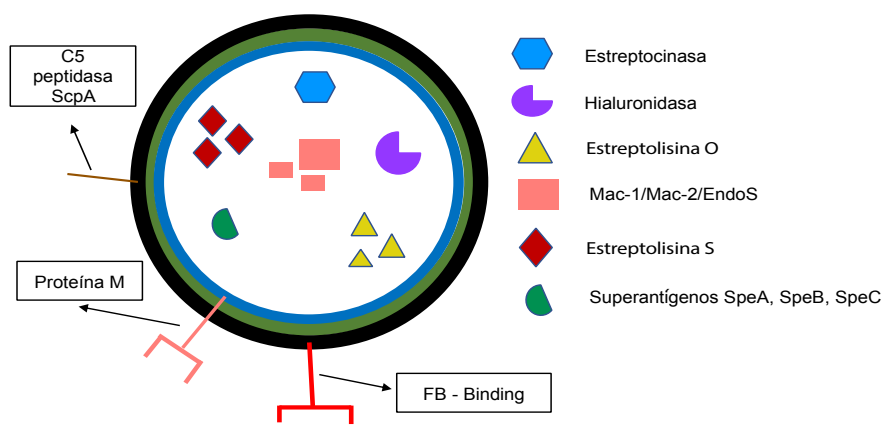

Figura 2. Streptococcus pyogenes factores de virulencia.

La bacteria cuenta hasta con 120 tipos de diferentes serotipos destacando el M1 y $M 3$, los cuales se encuentran hasta en el $50 \%$ de las infecciones severas estreptocóccicas como lo es la fascitis necrotizante y el síndrome del shock tóxico estreptocóccico; ${ }^{21}$ cada serotipo posee un número distinto de proteínas, por ejemplo: el serotipo M1/M49 tiene la proteína de unión a la fibronectina del grupo A estreptocóccico tipo
A (FbaA). ${ }^{21}$ El serotipo M1/M6/M12 tiene una proteína de unión a la fibronectina I $(\mathrm{Sfbl} / \mathrm{F1})^{21}$ y el serotipo M3 tiene la proteína de unión a la fibronectina del grupo A estreptocóccico tipo B (FbaB). ${ }^{21}$

En resumen, los serotipos conocidos de $S$. pyogenes que se relacionan principalmente con las lesiones cutáneas se describen en la tabla 1.

\section{Tabla I}

Principales serotipos de Streptococcus pyogenes relacionados con las lesiones cutáneas ${ }^{20,22,23}$

\begin{tabular}{|l|l|}
\hline \multicolumn{2}{|c|}{ SEROTIPO } \\
\hline M1 & Celulitis, síndrome del shock tóxico estreptocócicco, fascitis necrotizante \\
\hline M3 & Fascitis necrotizante, celulitis \\
\hline M5 & Celulitis, síndrome del shock tóxico estreptocócicco \\
\hline M6 & Fascitis necrotizante, celulitis en personas con uso de drogas intravenosas \\
\hline M28 & Fascitis necrotizante \\
\hline
\end{tabular}

\section{Mecanismos de evasión de la respuesta inmune}

Diversos estudios han demostrado que $S$. pyogenes al invadir una célula, puede inactivar el complemento. Diversos pacientes que desarrollan infecciones severas estreptocóccicas tienen una deficiencia o disminución de la proteína C3b del complemento, la cual juega un rol importante en la opsonización, estableciendo que los pacientes 
con diabetes y el paciente con enfermedad renal crónica (ERC) desarrollan con mayor frecuencia este tipo de infecciones. ${ }^{24}$

S. pyogenes cuenta con proteasas extracelulares como la exotoxina pirógena estreptocóccica tipo B (SpeB) la cual además de ser proteasa, se comporta como un superantígeno del cual se ha demostrado en estudios que es capaz de degradar el C3b en el suero humano además de inmunoglobulinas (IgG) y citosinas. ${ }^{24,25}$

Otro factor de virulencia igual de importante es la C5 peptidasas ( $\mathrm{ScpA}$ ), la cual se encarga de inactivar la fracción C5a del complemento, la cual está implicada en la quimiotaxis y el reclutamiento de los neutrófilos al sitio de infección y esto puede ser una de las razones por las cuales inicialmente en la zona de infección no se suele encontrar neutrófilos (figura 3). 25, 26

Otro mecanismo encontrado para evadir el sistema inmune es la secreción de varias enzimas entre las que destacan la enzima degradadora de inmunoglobulina $G$ de $S$. pyogenes (Mac-1/IdeS) y la endoglicosidasa de estreptococo (EndoS), las cuales son capaces de degradar IgG. ${ }^{24,27}$ Siendo Mac-1 la principal enzima de éstas en degradar el IgG con el mecanismo de unirse a esta inmunoglobulina provocando una separación proteolítica en el segmento cristalizable inhibiendo de esta forma la unión del complemento y el reconocimiento de la fracción Fc del anticuerpo (figura 3). ${ }^{28}$

\section{MECANISMO DE EVASIÓN DEL SISTEMA INMUNE}

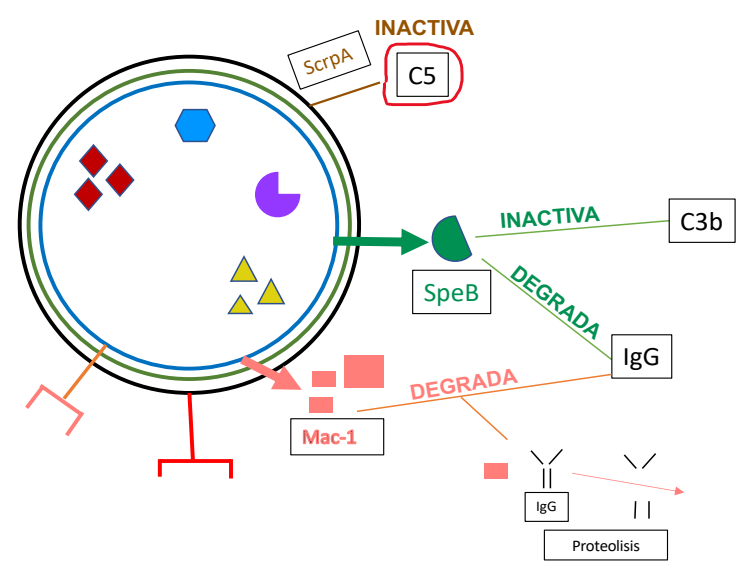

Figura 3. Mecanismo de evasión del sistema inmune, S. pyogenes.

\section{Mecanismos de lesión de la piel}

Una vez dentro de la célula y al haber inhibido al sistema inmune del huésped, continúa el proceso de patogénesis mediante la secreción de sus toxinas y otras enzimas entre las cuales cabe resaltar: la estreptocinasa, enzima proteolítica que transforma el plasminógeno a plasmina; de esta forma, propicia un estado anticoagulante; ${ }^{2}$ la hia- luronidasa, que degrada el ácido hialurónico del tejido conectivo; ${ }^{2}$ la estreptolisina $\mathrm{O}$ que es una citolisina dependiente de colesterol, la cual forma poros en las membranas celulares e inhibe la maduración de los autofagosomas y con ello la fagocitosis; ${ }^{18}$ la estreptolisina $S$ que es una toxina reconocida como una de las principales claves de la patogenia cutánea, ya que no sólo produ- 
ce la beta hemólisis de los eritrocitos, si no también en el rompimiento de las uniones intracelulares en la piel, además de provocar apoptosis de los macrófagos, neutrófilos y en últimos estudios se ha demostrado que también está involucrada en la necrosis de los queratinocitos. ${ }^{28}$ En este estudio se encontró que esta toxina acelera la apoptosis de los queratinocitos después de 6 horas de infección epitelial. ${ }^{28}$ Los queratinocitos son las células principales de la piel presentes desde la capa basal hasta la capa córnea, por lo que su destrucción representa una importante entrada para infecciones más profundas y aparición de bacteremias. ${ }^{29}$

La toxina tiene acción sobre la vía de señalización fosfatidilinositol 3 cinasa y la proteín-cinasa B (PI3k/Akt 1). Esta ruta de forma natural activa la proteín-cinasa B (Akt) la cual fosforila a la caspasa-9 inhibiendo la apoptosis en las células; la estreptolisina $S$ estaría regulando de forma negativa esta ruta, es decir, promoviendo la apoptosis de los queratinocitos. ${ }^{30}$

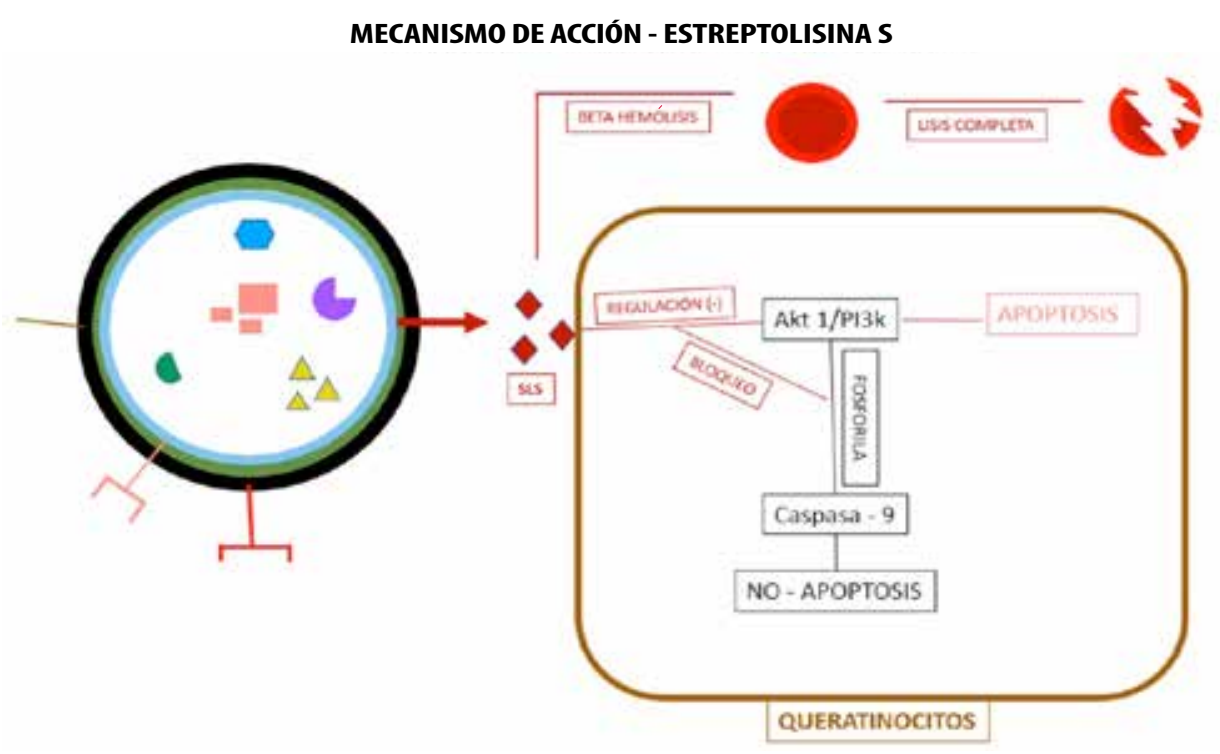

Figura 4. Mecanismo de acción de la estreptolisina S por S. pyogenes.

Superantígenos SpeA, SpeB, SpeC: son pequeñas proteínas no glicosiladas, estas proteínas poseen sitios de unión de zinc los cuales representan sitios de alta afinidad para los receptores $\mathrm{MHC}$ de clase $2 .{ }^{31} \mathrm{Su}$ importancia recae en la sobreexpresión de la respuesta inmunitaria en el caso de esta bacteria como síndrome del shock tóxico estreptocóccico y la fascitis necrotizante; ahora bien, de forma normal, al interactuar un antígeno con el MHC clase 2, se llega a estimular hasta el $0.01 \%$ de las células
T para que den una respuesta óptima al patógeno a tratar mientras que los superantígenos estimulan del $25 \%$ al $30 \%$; en consecuencia, se secretan de forma masiva las citosinas inflamatorias interleucina 2 (IL2), factor de necrosis tumoral (TNF) e interferón gama (INF $\gamma$ ). Esto conduce a que la persona presente un dolor desproporcional a la herida/traumatismo (en el caso de la existencia de ésta) así como la alta zona eritemato-edematosa y las ampollas (figura 5). ${ }^{25,32-34}$ 


\section{MECANISMO DE ACCIÓN - SUPERANTIGENOS}

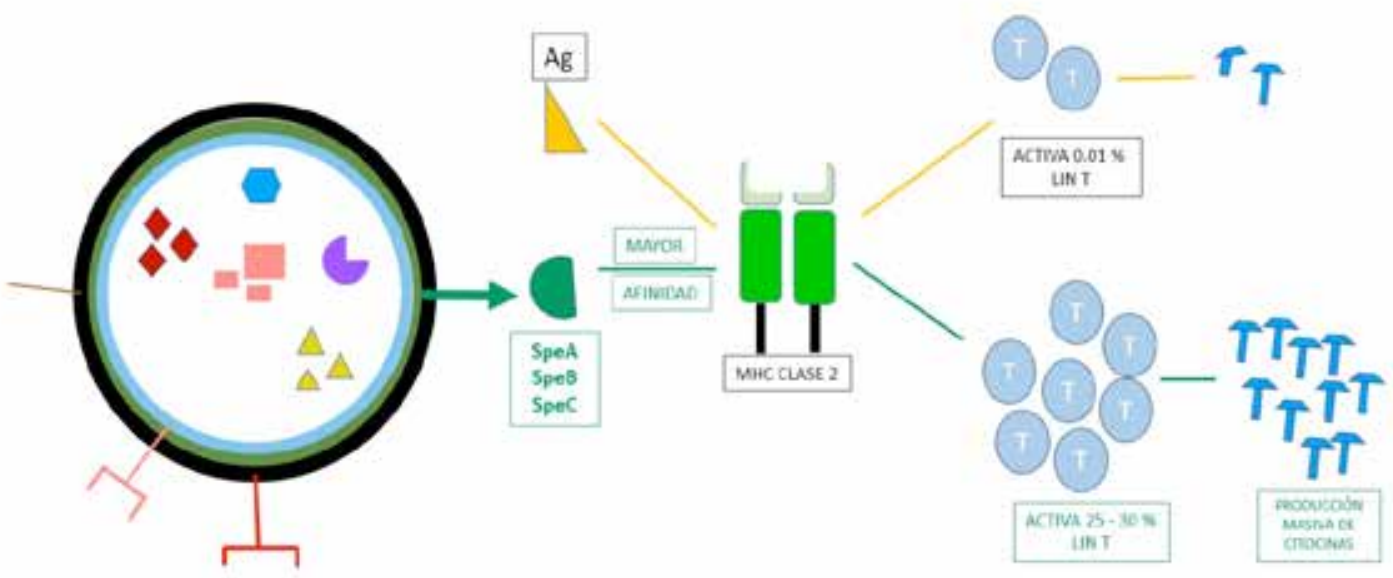

Figura 5. Mecanismo de acción de los superantígenos por S. pyogenes.

\section{Tipos de lesiones cutáneas}

Las principales lesiones cutáneas generados por S. pyogenes son: la celulitis, la erisipela y la fascitis necrotizante.

El proceso fisiopatológico de las lesiones causadas por $S$. pyogenes comienza desde que se tiene una herida, éstas pueden ser tan pequeñas como un mal rasurado, una tiña de los pies hasta quemaduras o heridas grandes, el estado inmunológico juega un papel sumamente importante aquí ya que sin un sistema inmune competente $S$. pyogenes tiene no sólo una vía de entrada libre (herida) si no también un sistema incapaz de eliminarlo dándole la facilidad de secretar todos sus factores de virulencia.

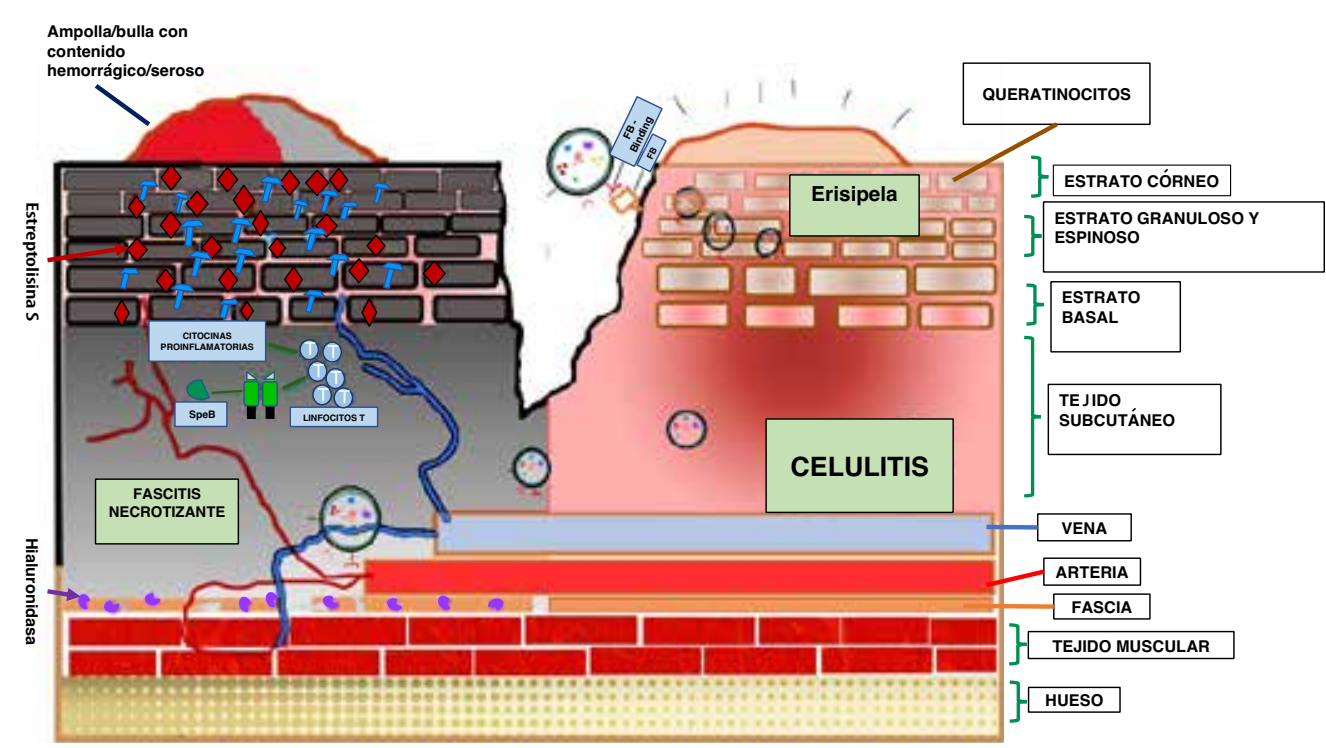

Figura 6. Mecanismo fisiopatológico de la erisipela, celulitis y fascitis necrotizante. 
Dentro de la patogénesis de las lesiones cutáneas se deben destacar los serotipos M1, M3 y M6, aislados de la mayoría de las lesiones cutáneas -erisipela, celulitis y fascitis necrotizante- en diversos estudios (figura 6). $18-21$

- El serotipo M1 se une a la fibronectina gracias a su que cuenta en su arsenal de proteínas con la fibronectina del grupo A estreptocóccico tipo A (FbaA). Este factor, además, le permite la evasión del sistema inmune ya que se ha visto que inhibe el depósito y activación del factor $\mathrm{C} 3 \mathrm{~b}$ del complemento. Este serotipo cuenta del mismo modo con la proteína de unión a la fibronectina I (Sfbl/F1), la cual también interfiere con el depósito de la fracción C3b del complemento. Ésta interactúa con las integrinas de los queratinocitos, enviando una señal para digerir a la bacteria. Cabe mencionar que posiblemente también el serotipo M6 podría tener esta proteína ya que se ha encontrado en diversos aislamientos de proteínas de éste. ${ }^{18,19}$

- En el serotipo M3 no se encuentra fibronectina del grupo A estreptocóccico tipo $A$ así como la proteína de unión a la fibronectina tipo I. Su unión a la fibronectina se encuentra mediada por la proteína de unión a la fibronectina del grupo A estreptocóccico tipo B (FbaB) la cual, además de permitir la unión, le concede la entrada a la célula mediante un mecanismo de "zipper/cremallera". ${ }^{21}$ Es importante señalar que los serotipos M1 y M3 poseen mayores mecanismos de virulencia ya que evaden al sistema inmune de la siguiente forma: inhiben el depósito del $\mathrm{C} 3 \mathrm{~b}$, inactivan la fracción $\mathrm{C} 5$ del complemento mediante el ScrpA; además, los superantígeno SpeB y las proteínas Mac-1 degradan al IgG. ${ }^{25}$

La erisipela es una forma de infección superficial donde la infección por $S$. pyogenes se limita a las capas superficiales de la epidermis y la dermis. Las manifestaciones clínicas (zona eritematosa limitada, con lesiones de aspecto circular y brillante por edema) estarían generadas por el inicio de la respuesta innata, donde el patógeno ya ha sido detectado con la consecuente respuesta inflamatoria y secreción de citocinas, además de toxinas y enzimas, como la hialurodinasa generadas por el S. pyogenes. ${ }^{18}$

La progresión de erisipela a una lesión por celulitis es generada por la llegada del $S$. pyogenes al tejido subcutáneo donde principalmente se encuentra el tejido adiposo y los vasos linfáticos. En este sitio se produce un proceso inflamatorio mucho mayor que en la erisipela, se pierde la limitación y forma circular de la lesión eritematosa inicial.

Para los pacientes con DM es más sencillo progresar y generar un cuadro de celulitis o fascitis necrotizante. Entre los factores involucrados destacan la falta de expresión de MHC tipo 1 en sus células mieloides y con ello la falta de actividad de los linfocitos T CD8. De esta forma, las células infectadas no se reconocen y no pueden terminar en un proceso apoptótico. Otros factores, como la disminución de la fracción C4 del complemento y la disminución en la quimiotaxis de las células polimorfonucleares generan una alteración y disminución en la respuesta inmune a $S$. pyogenes dándole mayor oportunidad a la bacteria de seguir su curso natural en la infección. 7,8,35

En el tejido subcutáneo, S. pyogenes secreta hialuronidasa, permitiendo la degradación de matriz intersticial hasta llegar a la fascia; a su vez, la estreptolisina S se encargaría de provocar la muerte de un número considerable de queratinocitos, produciendo el aspecto necrótico de la piel en la fascitis necrotizante. ${ }^{28,30}$

El superantígeno $S p e B$, debido a su gran afinidad por los receptores $\mathrm{MHC}$ de clase 2, favorecerían la secreción de manera masiva de citocinas inflamatorias, dando lugar a la aparición de una gran zona de inflamación cutánea, produciendo ampollas con contenido seroso $y$, de manera progresiva, un contenido seroso/hemorrágico; la aparición de dolor desproporcional a la herida tam- 
bién es consecuencia de la secreción de citocinas. Este síntoma se presenta primordialmente en caso de fascitis necrotizante. El tiempo de aparición de estas lesiones y manifestaciones es determinado por la respuesta inmunológica del huésped, y pude variar de horas a días o semanas (figura 7)..$^{35}$

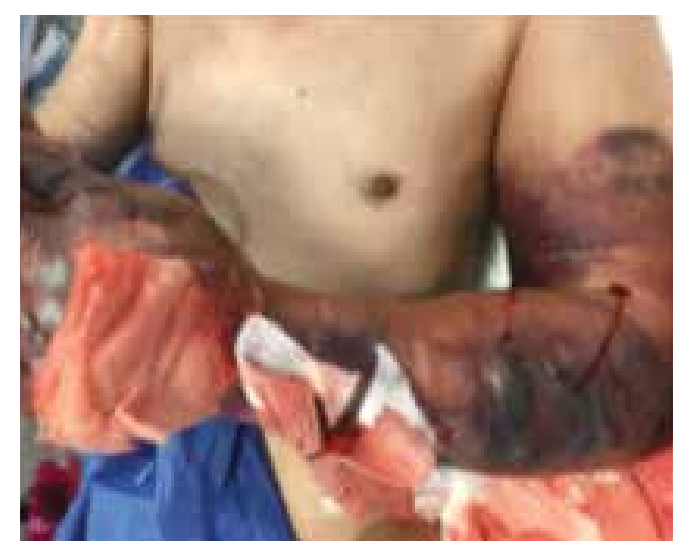

Figura 7. Hombre de 52 años con antecedentes de diabetes mellitus con diagnóstico de fascitis necrotizante a las 24 horas de sufrir una herida con un clavo en el tercer dedo de la mano izquierda. ${ }^{38}$

\section{Características clínicas de las lesiones cutánea secundarias a infección por S. pyogenes}

\section{Erisipela}

La erisipela se caracteriza por manifestarse como una placa eritematoedematosa, brillante y dolorosa, acompañada de fiebre y síntomas generales. Puede aparecer en cualquier zona del cuerpo donde se encuentre la lesión o herida que permite la entrada a la piel lesionada. Los sitios de mayor afección son las piernas, dorso de los pies y cara; en algunos casos, la persona puede presentar fiebre de $38^{\circ} \mathrm{C}$ a $40^{\circ} \mathrm{C}$ antes de que aparezca la lesión cutánea y ésta se puede diferenciar de una celulitis gracias a las siguientes características (figura 8): 38,39

a. Las lesiones toman un aspecto circular alrededor del foco de la lesión.

b. Bordes limitados.

c. Tienen un aspecto brillante edematoso y eritematoso.

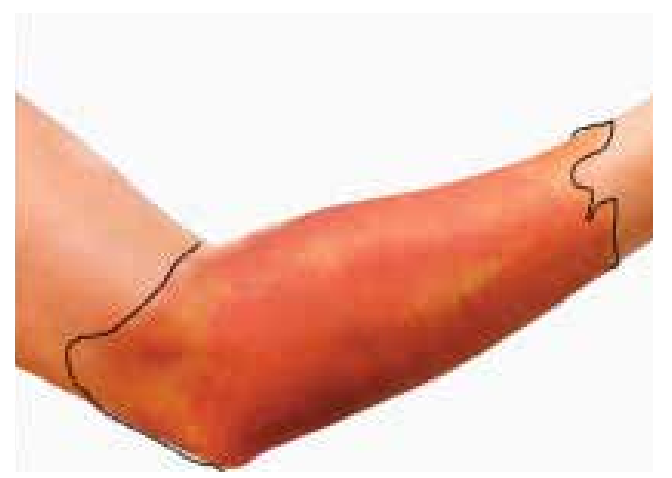

Figura 8. Erisipela, se puede observar una placa eritematosa, edematosacon límites bien determinados con aspecto brillante. ${ }^{36}$

\section{Celulitis}

Al igual que la erisipela, la celulitis puede manifestar síntomas generales antes de que se den las lesiones cutáneas. También pue- 
de aparecer en cualquier parte del cuerpo con predominio en piernas y cara, el área afectada se manifiesta como una zona eritemato-edematosa, dolorosa y caliente; el área de la lesión no tiene límites definidos y no posee un aspecto circular de la lesión (figura 9). 39,40

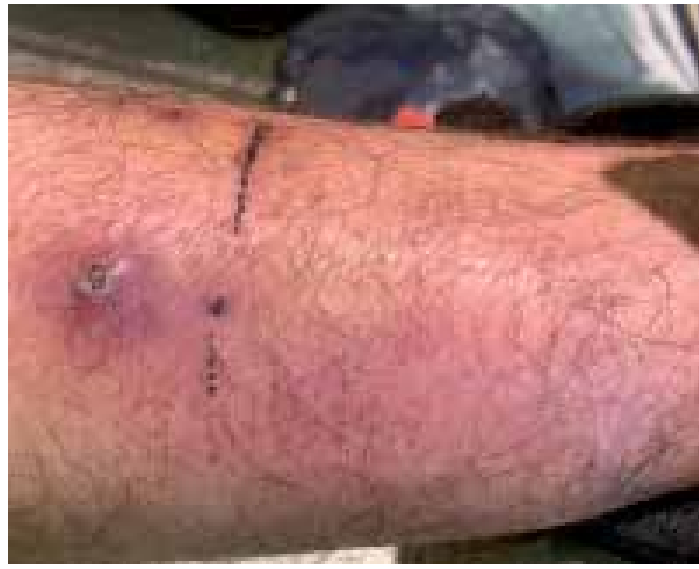

Figura 9. Celulitis infecciosa en miembro inferior izquierdo, se puede observar una placa eritematosa, edematosa con límites mal definidos.

\section{Fascitis necrotizante}

La fascitis necrotizante suele ser, en mayor medida, una infección polimicrobiana, pero también puede darse el caso de una infección monomicrobiana, resaltando entre las bacterias involucradas a S. pyogenes. La lesión se caracteriza por una rápida necrosis del tejido subcutáneo y la fascia, con una mortalidad que varía desde el $20 \%$ hasta el $60 \%$, siendo la DM el principal factor de mortalidad (figura 7 y 9). ${ }^{38}$ La lesión comienza con una zona eritematosa con hiperalgesia cutánea, pudiéndose confundir con una celulitis infecciosa; sin embargo, el dolor de la fascitis necrotizante es desproporcional con la herida en cuestión siendo el motivo principal por el que las personas acuden a recibir atención médica. ${ }^{40}$

Posteriormente comienzan a aparecer de forma progresiva ampollas/bullas, las cuales en su interior tendrán un fluido seroso y, tardíamente, el fluido será hemorrágico. Es importante diferenciar que los microorganismos anaerobios generarían gas en las ampollas/bullas. ${ }^{40}$

En este punto ya no sólo tendrán predominio las manifestaciones cutáneas si no se recibe un tratamiento adecuado: la persona puede desarrollar un estado de shock séptico o el síndrome del shock tóxico caracterizado por hipotensión, hipoxemia y falla orgánica múltiple. ${ }^{41}$

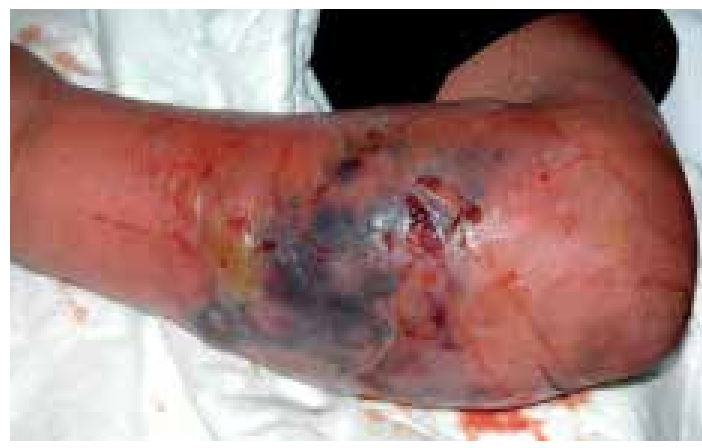

Figura 9. Fascitis necrotizante en miembro superior izquierdo, se logra ver un área eritematosa, necrótica con edema y con la presencia de bullas y ampollas hemorrágicas. ${ }^{29}$

\section{Conclusiones}

Las lesiones cutáneas por $S$. pyogenes en México se encuentran en aumento, tanto en número de casos, como en mortalidad asociada. Estos factores están relacionados con el aumento de personas diagnosticadas con DM y la falta de control metabólico ade- 
cuado. Es importante reconocer algunos mecanismos de inmunosupresión asociados a DM y la interacción con los factores asociados a la virulencia de agentes bacterianos dentro del proceso de infección. S. Pyogenes pertenece a la microbiota transitoria de la epidermis y mucosas de todas las personas. Si se favorece la entrada, colonización y se tienen condiciones óptimas de inmunosupresión — como en caso de DM y $\mathrm{VIH}$ se generarán lesiones cutáneas como erisipela, celulitis y fascitis necrotizante en estas poblaciones. El presente artículo pretende mejorar el conocimiento fisiopatológico de los principales factores de virulencia del $S$. Pyogenes y los mecanismos de producción de las lesiones cutáneas, así como familiarizar al lector con la identificación clínica correcta de estas lesiones para su oportuna diferenciación y obtención de un diagnóstico que mejore el pronóstico del paciente.

\section{Bibliografía}

1. Brooks G, Carroll K, Butel J, Morse S, Mietzner T. Jawetz, Melnick y Adelberg Microbiología Médica. 27th ed. New York: Mc Graw Hill; 2020.

2. Murray $P$, Rosenthal K, Pealler M. Medical Microbiology. 9th ed. Amsterdam: Elsevier; 2020.

3. Zoszoli A, Meyer B, Adair E, Torgov V, Veselovsky V, Danilov $L$ et al. Group A, B, C, and C Streptococcus Lancefield antigen biosynthesis is initiated by a conserved ?-D-GICNAC-?-1,4-L-rhamnosyltransferase. Journal of Biological Chemistry [Internet]. 2020 [cited 9 September 2020];295(16):1. Available from: https://www.jbc.org/ content/early/2020/03/04/jbc.RA119.011851.abstract.

4. Lopardo H, Predari S, Vay C. Manual de Microbiología Clínica De La Asociación Argentina De Microbiología [Internet]. 1st ed. Argentina: Universidad Nacional de la Plata; 2017 [cited 16 August 2020]. Available from: http://1url. pw/e1LOc

5. Espadas D, Flor E, Borrás R, Poujois S, Muñoz J. Infección por estreptococo pyogenes en la edad pediátrica: desde faringoamigdalitis aguda a infecciones invasivas. Anales de Pediatría [Internet]. 2018 [cited 16 August 2020];88:7581. Available from: https://www.sciencedirect.com/science/article/pii/S1695403317300565.

6. Watts V, Balasegaram S, Brown C, Mathew S, Mearkle R, Ready D et al. Increased Risk for Invasive Group A Streptococcus Disease for Household Contacts of Scarlet Fever Cases, England, 2011-2016. Emerging Infectious Diseases [Internet]. 2019 [cited 16 August 2020];25(3):529, 534. Available from: https://wwwnc.cdc.gov/eid/article/25/3/18-1518_article.

7. Casqueiro J, Casqueiro J, Alves $C$. Infections in patients with diabetes mellitus: A review of pathogenesis. Indian Journal of Endocrinology and Metabolism [Internet]. 2012 [cited 20 August 2020];16(7):27-28. Available from: https:// www.ncbi.nlm.nih.gov/pmc/articles/PMC3354930/.

8. Machado L, Montano M, Dimakis D. Diabetes mellitus y su impacto en la etiopatogenia de la sepsis. Acta médica Grupo Ángeles [Internet]. 2020 [cited 21 August 2020];15(3):208 - 212. Available from: http://www. scielo.org.mx/scielo.php?script=sci_arttext\&pid $=$ S187072032017000300207\&lng=es\&tlng=es.

9. Secretaría de Salud. Sistema de vigilancia epidemiológica hospitalaria de diabetes mellitus tipo 2, Boletín de Diabetes tipo 2 cierre 2019. Gobierno de México; 2020 p. 13, 21.

10. Secretaría de Salud. Sistema de vigilancia epidemiológica hospitalaria de diabetes mellitus tipo 2, Boletín de Diabetes tipo 2 cierre 2018. Gobierno de México; 2019 p. 11, 24.

11. Secretaría de Salud. Sistema de vigilancia epidemiológica hospitalaria de diabetes mellitus tipo 2, Boletín de Diabetes tipo 2 cierre 2017. Gobierno de México; 2018 p. 8, 22.

12. Secretaría de Salud. Sistema de vigilancia epidemiológica hospitalaria de diabetes mellitus tipo 2, Boletín de Diabetes tipo 2 cierre 2016. Gobierno de México; 2017 p. 10, 23.

13. Secretaría de Salud. Sistema de vigilancia epidemiológica hospitalaria de diabetes mellitus tipo 2, Boletín de Diabetes tipo 2 cierre 2015. Gobierno de México; 2016 p. 5, 22.

14. Secretaría de Salud. Sistema de vigilancia epidemiológica hospitalaria de diabetes mellitus tipo 2, Boletín de Diabetes tipo 2 cierre 2014. Gobierno de México; 2015 p. 2, 16.

15. Secretaría de Salud. Sistema de vigilancia epidemiológica de VIH, Datos históricos VIH-SIDA 1983-2019. Gobierno de México; 2020 p. 7, 27.

16. Sánchez L, Anco K. Cellulitis and erysipela. Dermatología Peruana [Internet]. 2020 [cited 19 August 2020];26(1):1314. Available from: http://www.dermatologiaperuana.pe/ assets/uploads/revista_R5Dd_Rev_Dermatol 26-1.pdf.

17. Wallace $H$, Perera $\bar{T}$. Necrotizing Fasciitis [Internet]. StatPearls. 2020 [cited 9 March 2021]. Available from: https://www.ncbi.nlm.nih.gov/books/NBK430756/\#_ NBK430756_pubdet

18. Yamaguchi $\bar{M}$, Terao $Y$, Kawabata $S$. Pleiotropic virulence factor - Streptococcus pyogenes fibronectin?binding proteins. Cellular Microbiology [Internet]. 2012 [cited 20 August 2020];15(4):503 - 504, 506 - 507. Available from: https://onlinelibrary.wiley.com/doi/full/10.1111/ cmi.12083.

19. Stetzner Z, Li D, Feng W, Liu M, Lui G, Wiley J et al. Serotype $M 3$ and M28 Group A Streptococci Have Distinct Capacities to Evade Neutrophil and TNF-? Responses and 
to Invade Soft Tissues. Public Library of Sciencie [Internet]. 2015 [cited 31 August 2020];10(6):1-2, 7. Available from https://journals.plos.org/plosone/article?id=10.1371/ journal.pone.0129417.

20. Gonzáles $M$, Alonso $M$. Invasive Streptococcus pyogenes infections (2011-2018): EMM-type and clinical presentation. Anales de Pediatría [Internet]. 2020 [cited 30 August 2020];92(6):353 - 354. Available from: https://www.analesdepediatria.org/en-invasive-streptococcus-pyogenes-infections-20112018--articulo-resumen-S2341287920300703.

21. Ochel A, Rohde $M$, Chhatwal G, Talay S. The $M 1$ protein of Streptococcus pyogenes triggers an innate uptake mechanism into polarized human endothelial cells. Journal of Innate Immunity [Internet]. 2014 [cited 17 August 2020];6(5):585 - 586. Available from: https://www.karger.com/Article/FullText/358085.

22. Metzgar D, Zampolli A. The M protein of group A Streptococcus is a key virulence factor and a clinically relevant strain identification marker. Virulence [Internet]. 2011 [cited 21 August 2020];2(5):402 - 408. Available from: https://pubmed.ncbi.nlm.nih.gov/21852752/.

23. Terao $Y$. The virulence factors and pathogenic mechanisms of Streptococcus pyogenes. Journal of Oral Biosciences [Internet]. 2012 [cited 17 August 2020];54(2):96-99. Available from: https://www.sciencedirect.com/science/article/ pii/S1349007912000497.

24. Laabei $M$, Ermert D. Catch Me if You Can: Streptococcus pyogenes Complement Evasion Strategies. Journal of Innate Immunity [Internet]. 2019 [cited 17 August 2020];11(1):3-9. Available from: https://www.karger. com/Article/Abstract/492944.

25. Barnett T, Cole J, Rivera T, Henningham A, Paton J, Nizet $\mathrm{V}$ et al. Streptococcal toxins: role in pathogenesis and disease. Cellular Microbiology [Internet]. 2015 [cited 17 August 2020];17(12):1721，1723，1725, 1726, 1728 1729. Available from: https://onlinelibrary.wiley.com/ doi/10.1111/cmi.12531.

26. Rammingen V. Streptococcal IdeS and Its Impact on Immune Response and Inflammation. Journal of Innate Immunity [Internet]. 2012 [cited 17 August 2020];4(2):133134. Available from: https://pubmed.ncbi.nlm.nih gov/22248585/.

27. Zhu L, Olsen R, Lee J, Porter A, DeLeo F, Musser J. Contribution of Secreted NADase and Streptolysin $O$ to the Pathogenesis of Epidemic Serotype M1 Streptococcus pyogenes Infections. American Journal of Pathology [Internet]. 2017 [cited 17 August 2020];187(3):607. Available from: https://pubmed.ncbi.nlm.nih.gov/28034602/.

28. Flaherty R, Puricelli J, Higashi D, Park C, Lee S. Streptolysin $S$ Promotes Programmed Cell Death and Enhances Inflammatory Signaling in Epithelial Keratinocytes during Group A Streptococcus Infection. Infection and Immunity [Internet] 2015 [cited 17 August 2020];83(10):4121-4124. Available from: https://pubmed.ncbi.nlm.nih.gov/26238711/.

29. Bolognia J, Schaffer J, Cerroni L. Dermatology. 4th ed. Amsterdam: Elsevier; 2017.

30. Pinzón $C$, Serrano $M$, Sanabria $M$. Papel de la vía fosfatidilinositol 3 kinasa (PI3K/Akt) en humanos. Revista Ciencias de la Salud [Internet]. 2009 [cited 18 August
2020];7(2):48, 50. Available from: http://www.scielo.org. $\mathrm{co} /$ scielo.php?script $=$ sci arttext\&pid $=\mathrm{S} 1692$ $72732009000200007 \& \operatorname{lng}=$ en\&tlng $=$ es.

31. Li H, Zhao Y, Li Z, Eisele L, Mourad W. Zinc induces dimerization of the class II major histocompatibility complex molecule that leads to cooperative binding to a superantigen. Journal of Biological Chemestry [Internet]. 2007 [cited 18 August 2020];282(9):5991. Available from: https://www. ncbi.nlm.nih.gov/pmc/articles/PMC3924565/.

32. Rohde M, Cleary P. Proft T. Frasser J. Streptococcus pyogenes Basic Biology to Clinical Manifestations [Internet] Oklahoma: University of Oklahoma Health Sciences Center Oklahoma City (OK); 2017 [cited 18 August 2020]. Available from: https://www.ncbi.nlm.nih.gov/books/ NBK333420/.

33. Punt J, Owen J, Stranford S. Inmunología de Kuby. 7th ed. New York: Mc Graw Hill; 2014.

34. Davies F, Olme C, Lynskey N, Turner C, Sriskandan S. Streptococcal superantigen-induced expansion of human tonsi $T$ cells leads to altered $T$ follicular helper cell phenotype, B cell death, and reduced immunoglobulin release. Clinical and Experimental Immunology [Internet]. 2018 [cited 18 August 2020];197(1):1-2. Available from: https://www. biorxiv.org/content/10.1101/395608v1.article-info.

35. Ferrer $Y$, Morejón Y. Fascitis necrosante. Actualización del tema. MediSur [Internet]. 2018 [cited 19 August 2020];16(6):921, 923. Available from: http://scielo.sld. $\mathrm{cu} /$ scielo.php?script $=$ sci_arttext $\&$ pid $=$ S1727-897X20180 00600015\&lng $=$ es\&tlng $=$ es.

36. Michael $Y$, Shaukat N. Erysipelas [Internet]. Ncbi.nlm.nih. gov. 2020 [cited 19 August 2020]. Available from: https:// www.ncbi.nlm.nih.gov/books/NBK532247/.

37. Moreno J, Muzquiz A, Hernández M, Bonifaz A. Fascitis necrosante. Abordaje diagnóstico y terapéutico. Dermatología Revista Mexicana [Internet]. 2020 [cited 19 August 2020];64(4):449-450. Available from: https://dermatologiarevistamexicana.org.mx/article/fascitis-necrosanteabordaje-diagnostico-y-terapeutico/.

38. Palmieri $O$, Corti $M$, Laube $G$, Nergoni R. Enfermedades Infecciosas. 3rd ed. New York: Mc Graw Hill; 2009.

39. Roda A, Pinto A, Filipe A, Freitas J, Filipe P. Clinical and Laboratory Factors Associated with Prolonged Hospital Stay among Patients with Cellulitis/Erysipelas. Acta Médica Portuguesa [Internet]. 2019 [cited 19 August 2020];32(6):448-449. Available from: https://pubmed. ncbi.nlm.nih.gov/31292026/.

40. Fumis $M$, Bidabehere $M$, Moyano $Y$, Sardoy $A$, Boldrini $M$, Pinardi $B$ et al. Fascitis necrotizante por Streptococcus pyogenes: a propósito de un caso. Revista de la Facultad de Ciencias Médicas de Córdoba [Internet]. 2020 [cited 19 August 2020];73(3):283-284. Available from: https://revistas.psi.unc.edu.ar/index.php/med/article/view/16627.

41. Gonzáles N, Vega R, Romero L, Gómez V. Síndrome de choque tóxico por Streptococcus pyogenes: reporte de caso y revisión de la literatura. Revista Latinoamericana de Infectología Pediátrica [Internet]. 2020 [cited 19 August 2020];30(3):117, 119-120. Available from: https://www.medigraphic.com/cgi-bin/new/resumen. cgi?IDARTICULO=75094 